\section{ON A SIMPLE METHOD OF MEASURING THE ALKALINITY OF THE BLOOD.}

By A. E. WRIGHT, M D. Dub.,

PROFESSOR OF PATHJLOGY, ARMY MEDICAL SCEOOL, NETLEY.

\section{Preliminary Considerations.}

BEFORE embarking upon the description of the method of measuring the alkalinity of the blood which is to be dealt with in this paper it will be profitable briefly to discuss the following series of questions :-

1. What is the meaning of the term "alkalinity"?-It is not by any means sufficiently realised that the term alkalinity is an eminently ambiguous term. It is a term which may at one moment be employed to denote the quality of consisting of, or containing in solution, a free base, while at another moment it is employed to denote quite a different thingto wit, the quality of consisting of, or containing in solution, bases which are only very loosely combined with acids.

2. In what sense is the blood an alkaline fluid? - The blood is an alkaline tluid not in the first, but only in the second sense of the term. It is, in other words, an alkaline fluid only in the sense of being a fluid which contains bases which are conjugated with very weak acids.

3. What is our criterion of the alkalinity of a fluid which does not contain free bases? - It will be obvious to consideration that the only pessible criterion of alkalinity which is applicable in the case of a flaid which dses not contain free bases is the capacity of such a fluid for yielding up bases to weak acids. This capacity of yielding up bases to weak asids is preciety the quality which is tested by indicators. It follows therefore that our criterion of the alkalinity of the blood is the reaction of blood to an indicator.

4. What is our measure of the alkalinity of the fluid which does not contain free salts. - It is a corcllary from what has been said above that we can obtain a measure of the alkalinity of such a flaid as the blood by determining what amount of acid addition will tie up all its bases in combinations from which indicators (or, as we may more properly call them, "indicator-acids") will be unable to withdraw them.

The theoretical groundw ork of all hæmalkimetrical methods is contained in the answers to these questions. Taking our stand upon this we may pass on to consider what is the most suitable variety of indicator, and what is the most suitable variety of tritrating acid to employ in our bæmalkimetrical observations.

Selection of a suitable variety of indicator. - Indicator-acids, like other acids, differ amongst themselves in their avidity for bases. Some indicatore, and litmus is a good example of such an indicator, are able to withdraw bases, not only from the normal salts of weak acids (e.g., from $\mathrm{Na}_{2} \mathrm{CO}_{3}$ and $\mathrm{Na}_{3}$ $\mathrm{P0}$ ), but also from certain chemically acid saits (e.g., from $\mathrm{NaHCO}_{3}$ and $\mathrm{Na}_{2} \mathrm{HPO}_{4}$ ). Othr $\mathrm{r}$ indicators, such, for instance, as phenolphtha!ein, are indeed able to withdraw bases from the normal salts of weak acids, but they are quite unable to withdraw bases from any chemically acid salts. It will be manifest on consideration of these facts that when we are testing a fluid which, like the blood, contains chemically acid salts in solution, it will not be a matter of indifference to our results whether we employ litmus or phenolphthalein as our indicator. On the contrary, the results which we obtain with the one variety of indicator will differ, not only quantitatively, but also qualitatively, from those which we obtain with the other variety of indicator. We sball find, for instance, that normal blood is always definitely alkaline to litmus, whereas it is almost always neutral or acid to phenolphthalein. We shall find, in other words, that litmus is always able to withdraw bases from normal blood, whereas phenolphthalein is generally unable to do so. In the light of this fact it will be manifest that when we propose to ourselves to measure the alkalinity of the blood we are in point of fact proposing to ourselves to measure the capacity of the blood for yielding up bases to one particular variety of indicator-to wit, to free (i.e, red) litmus. Onr question as to what is the most suitable variety of indicator to emcloy for hæmalkimetrical purposes is thus in some measure resolved.

$1 \mathrm{C}$ nsideration will show that these indicators a"e in reality acid substinces which differ from otber acid substances "nly in possessing the property of combining with ba:es to form distinctively coloured
The most suitable indicator will be a red or neutral tinted litmus paper. ${ }^{2}$ We have still to consider the question of the sensitiveness of our indicator.

Selestion of a sufficiently sensitive sample of red litmus paper. - It will be obvious to reflection that inasmuch as red litmus is prepared by liberating the litmus acid from its salts by an excess of added acid, ordinary red litmus paper will contain traces of free acid which will seriously interfere with its sensitiveness to the presence of bases. In view of this fact, every litmus paper which is employed in hæmalkimetrical observations ought previously to be tested as to its sensitiveness. A good sample of red or neutral tinted litmus paper ought to show a distinct and immediate colour change when it is dipped into a forty-fold diluted normal human serum.

Seleotion of a suitable variety of tritrating acid.-It has been customary to employ solutions of organic acids (solutions, for instance, of oxalic, citric, or tartaric acids) for hæmalkimetrical purposes. The employment of these acids presents an undoubted advantage from the point of view of convenience of preparation. For standardised solutions of these acids can in each case be prepared by merely dissolving a weighed quantity of the particular acid in a measured quantity of water. On the other hand, the employment of these solutions of organic acids is open to serious objection, inasmuch as solutions of these organic acids become gradually weaker owing to the breaking down of these acids under the influence either of bacteria or of light and oxygen. We shall avoid this great source of fallacy in our mea:urements if we employ standardised solutions of sulphuric acid as our tritrating acid. These questions relative to the most suitable indicator and tritrating acid having been satisfactorily resolved we may pass on to consider the methods of dealing with practical difficulties which are associated first, with the presence of blood corpuscles in the blocd the alkalinity of which we desire to elicit, and, secondly, with the necessity of operating with minimal quantities of blood.

Methods of dealing with the diffioulties which are associated with the presence of red blood corpuscles in the blood.-Various mothods of dealing with these difficulties have been pro posed. Liebreich proposed to deal with these difficulties by impregnating slabs of alabaster or porous clay with litmus. The upper surface of these slabs was intended to hold back the red blood corpuscles while the plasma infiltrated into the substance of the slab. It was proposed to bring the colour change which occurred in the litmus into view by washing off the layer of red blood corpuscles wbich had deposited itself on the surface of the slab. Schäfer proposed to deal with the difficulty in a somewhat similar way. $\mathrm{He}$ coated over his litmus paper with a glaze which was designed to bold back all the red blood corpuscles while it remained perfectly permeable to the plasma. Lastly, Landois, Drouin, and others have dealt with the difficulty by diluting the blood with various salts solutions, and by allowing this diluted plasma which was thus obtained to purge itself of it red blood corpuscles by creeping up by capillarity into a strip of test-paper which was hung in to the containing vessel None of the se three methods appears to be well adapted to the quantitative determination of the alkalinity of the bloou, for by none of these methods can we effect that complete separation of the red blocd corpusc?es from the blood fluid which is absolutely essential to the accurate determination of the neutral point. In addition to being open to objection in this respect, the method which is employed by Landois and Drouin is open to the serious objection that the more the blood is diluted the more difficult does it become to determine the neutral point with accuracy.

Selection of a variety of blood fluid which shall be suitable for hamalkimetrical observations. - When we take the defects of the above-mentioned methods into consideration we begin to ask ourselves whether it is not permissible to dispense

2 Blue litmus paper might also be employed as an indicator inasmuch as it would indicate to us the point at which a surplus of tritrating acid has been added to the blood. But the use of blue litmus would be associated with a double disadvantage. In the firs place, when blue litmus is employed it is always a matter of som difficulty to distinguish the red colouration which is indicative of surplus of added acid from the red colouration which is referable to the presence of red blood corpuscles. In the sezond place, when blue itmus is employed it is diffeult to dit tinguish the fugitive red colouration whicb is produced by the carbonic acid which is evolved from the blood under the influence of the tritrating acid from the red coloura tion which is indicative of the presence of an excess of this tritrating acid. It is obvious that both these sources of fallacy are aroided by the employment of a red or neutral tinted litmus. 
with the immediate separation of the corpuscles from the blood-fluid, and to employ a blood-fluid from which the corpuscles have been separated by more effectual and more leisurely processes. We begin to ask ourselves, in fact, whether we may not employ for alkalimetrical purposes, either the perfectly corpuscle-free plasma, which may be obtained by allowing a decalcified blood to sediment in a capillary tube, or the perfectly corpuscle-free serum, which can be obtained by allowing the serum to exude from an ordinary blood-clot. As a matter of fact, an objection can be raised against the employment of either of these blood fluids. This objection is based upon the fact that the blood undergoes a gradual diminution in alkalinity after it has left the bloodvessels. It might, therefore, be urged that the alkalinity of the sedimented plasma or exuded serum would not be a correct measure of the alkalinity of the circulating blood. But considoration will show that this would not be a fatal objection to the utilisation of serum or sedimented plasma if it could be shown, first, that the serum and plasma ultimately come to a condition of stable equilibrium in the matter of alkalinity, and secondly, that the deficitive alkalinity of these blood fluids is a faithful index, or even a tolerably faithful index, of the alkalinity of the intravascular blood. Now it is practically certain that these conditions are actually realised. In the first place observations of Zuntz, Peiper, and Winternitz on the serum, and similar observations of my own on the plasma, bave shown that the serum and plasma arrive at a condition of stable equilibrium, within at any rate a few hours, after the blood has been withdrawn from the vessels. And, in the second place, observations of Drovin, which again have been supplemented by a considerable number of observations of my own, have shown that changes in the alkalinity of the circulating blood invariably manife:t themselves in changes in the alkalinity of the serum. In view of these facts, and of the fact that it is difficult, if not impossible, to determine with any degree of precision the neutral point of a bloodfluid which contains any red blood corpuscles, it will be obvious that we shall be able to obtain more trustworthy data with reference to the variations in the alkalinity of the blood if we employ, in lieu of the fresh plasma which is contaminated with red blood corpuscles, either the corpusclefree serum which exudes from a clot or the corpuscle-free plasma which can be obtained by the sedimentation of decalcified blood. Our simplest course-and in these matters the simplest course is always the best course-would appear to be to ersploy for all hæmalkimetrical purposes the serum which separates out from an ordinary blood clot.

Methods of dealing wath the difficulties nhich are associated with the necessity of operating with minimal quantities of blood-fluid.-The difficulties which are associated with the necessity of operating with minimal quantities of blood-fluid are generally got over by measuring off a series of very small samples of blood fluid into a series of accurately measured, and progressively increasing, quantities of the tritrating acid. The principles of this method cannot be improved upon. On the other hand, the procedure which is usually adopted may be improved upon in two particulars. In the first place, it is well, instead of measuring off the blood or blood fluid into many times its volume of the acid solution, to mix the blood fluid with not more than an equal quantity of the acid solution, and so to avoid unduly diluting it. In the second place, it is well in measuring out the required quantities of acid and blood fluid to employ thin-walled capillary pipettes ${ }^{3}$ (such as can be made by drawing out a piece of ordinary glass tubing in the flame) instead of the thick-walled capillary pipettes which are made out of thermometer tubing. The advantages which we gain by employing such thin-walled capillary pipettes are the following: (1) we can readily fill in these capillary pipettes by capillary attraction alone; (2) we do not lose any of our fluid by diffusion over the external surface of the pipette ${ }^{4}$; and (3) we can at any moment and without any trouble make and calibrate such pipettes for ourselves instead of having to purchase them from a glass-blower. We have now sufficiently

3 The method of making and calibrating these pipettes will be described below.

described will be obvious that this consideration is not of any great importance when, as, for instance, in the case of blond corpuscle estimations, we are measuring off a small quantity of blood into a much larger quantity of diluting fluid. On the other hand, wherever, as here, we are concern d to mix a small quantity of blood with an equally small quantity of a diluting fluid, it is essential to accuracy that we
should not lose any of our fiuid by its diffusion over the surface of the pipette. broken the ground, and may, therefore, pass on without further prologue to the description of the practical details of the method which has been foreshadowed above.

\section{Practical Details of the Method of Measuring THE ALKALINITY OF THE BLOOD.}

Preparation of the necessary apparatus.-The necessary apparatus is of the simplest kind. It consists of a couple of glass capsules for receiving the blood which is drawn off from the finger, of one or more capillary pipettes for measuring and mixing the serum with the tritrating acid, and of half-a-dozen watch-glasses. The blood capsules and the capillary pipettes ${ }^{\overline{5}}$ are made out by drawing out pieces of ordinary glass tubing (of, say, five-sixteenths of an inch diameter) after heating them in a flame.

Preparation of the necessary reagents.-The necessary reagents consist $(a)$ of a supply of a sufficiently sensitive red litmus paper; and $(b)$ of a series of dilutions of a standardised sulphuric acid. The preparation of a sufficiently sensitive red litmus paper presents no difficulity if we have at our disposal a satisfactory sample of blue litmus paper, such, for instance, as may be obtained from Grübler and Co., of Leipzig. This blue litmus paper is first to be steeped in a very weake.g., in a 1 in 20,000-solution of hydrochloric acid until it assumes a reddish colour. It is then to be carefully washed in distilled water until all traces of fresh acid are removed. Finally, it is to be dried in a hot-air chamber. A litmus paper which is thus prepared will be sensitive enough to respond with an immediate colour change when it is dipped into a twenty - to forty-fold dilution of normal buman serum. The weak solutions of standard acids which we require for purposes of tritration can be conveniently made by making a series of progressive dilutions of normal sulphuric acid. Five such solutions-to wit, a twenty-, thirty-, forty-, fiftyand sixty-fold dilution - of normal sulphuric acid will suffice for all ordinary purposes. From these, as will be presently explained, any further dilations which may be necessary may very easily be prepared.

Method of obtaining blood and serum for hemallimetrical purposes.-A sufticient supply of blocd can be obtained from a prick in the finger-tip. The most convenient implement to employ for drawing blood is a sharp-pointed steel nib one of whose points has been broken off. Blood in ample quantities can be obtained by twisting a handkerchief round the finger and making pressure from above downwards. The blood which is obtained in this manner is filled into one or two blood capsules. It may either be aspirated into the capsule, or-and this is the easier plan-the blood capsule may be held at a lower level than the drop of blood, and the blood may then be allowed to run in by gravity, aided by capillary attraction. In any case the capsule ought to be filled in in such a manner that one of its ends may remain perfectly free from blood. When the capsule has been filled in, one or both of its ends may be sealed up in the flame. The blood is then to be allowed to clot undisturbed. As soon as the clot has sufficiently retracted and the serum has attained its definitive alkalinity-i.e., after a period of from three to twenty-four hours-both ends of the capsule are broken off and the colourless serum ${ }^{7}$ is drawn off by inserting the end of the capillary pipette into the capsule.

Method of performing the tritration - Having obtained our serum, it will be convenient to draw off from the capsule at first only the quantity which will be needed for our first tritration. It will be well not to employ for this first tritration more than about a sixth of the whole available quantity.

5 A blood capeule consists of a piece of glass tubing which has been heated in two places about half an inch to an inch apart, and which has been drawn out at these places into a capillary tube. A capillary pipette consists of a piece of glass tubing which has been heated in one place, and which has been drawn out there into a capillary tube. It is convenient, but it is not essential, to form a mixing chamber upon the capillary pipette by heating and drawing out the tube again at the point where the wide tube begins to tail off into the capillary
stem.

6 If an ordinary blow-pipe flame or a Bunsen flame is not available the glass tubing can, in conformity with a suggestion which was made by Surgeon-Major Semple and myself, be satisfactorily drawn out applying a lighted match to a finely divided spray of methylated applying

7 The serum which is obtained in this manner will, in the large majority of cases, be perfectly free from blood-corpuscles. If, however, from them by a simple process of sedimentation. To accomplish this it from them by a simple process of sedimentation. To accomplish this it the bloody serum and to set it up on end for a few minutes in a testtube rack. 
If the total quantity which is available is a very small quantity we shall therefore employ an extremely fine capillary pipette. If, on the other band, we have a relatively considerable quantity of seram available we shall employ a capillary pipette of correspondingly larger bore. In any case, we shall do well to arrange matters so that the quantity of serum which we have at disposal for our first tritration shall occupy not less than 1-2 cm. of the stem of our capillary pipette. We may assume for the sake of fixing our ideas that it occupies a length of exactly $2 \mathrm{~cm}$. In such a case we make a mark with a red wax pencil upon the stem of the pipette at a point about $2 \frac{1}{5} \mathrm{~cm}$. above the end of the pipette. Having done this, we turn up the point of the pipette and allow the serum to run down the stem of the pipette up to the point which is indicated by the red pencil mark. The object of this manceuvre is to take an air.bubble index into the point of the pipette. Having done this, we proceed to dip the end of the capillary pipette into a drop of our twenty-fold dilution of normal sulphuric acid, and we allow the acid to run up into the stem of the pipette until the upper margin of the air-bubble which separates the serum from the acid just reaches the red mark upon the stem of our pipette. It is obvious that when the air-bubble index reaches this point the quantity of acid solution which has been run up into the stem of the pipette will be exactly equivalent to the amount of serum which was drawn off into the pipette. Having thus obtained exactly equal quantities of our serum and our tritrating acid, our next step will be to mix the contents of our capillary pipette. We can do this either by drawing up the contents of our capillary tubes two or three times through our mixing chamber, or, if our tube is unprovided with a mixing chamber, by blowing out the contents of our capillary pipette into a clean watch-glass and then re-aspirating them, after complete mixture bas been effected, into our pipette. Mixture of the contents of our pipette, having been accomplished, we have only to blow out the mixture of serum and tritrating acid in a series of separate drops on to the surface of a strip of our red litmus paper. In the case of the particular mixture we are considering-i.e., in the case of a mixture consisting of equal volumes of serum and a twenty-fold diluted normal sulphuric acid-we should almost certainly find that we had in our mixture an excess of acid. We should then proceed in a precisely similar manner to tritrate with each other equal volumes of serum and a thirtyfold diluted normal acid. If we were dealing with normal blood this mixture might or might not have a slightly acid reaction. If this mixture proved to be still acid we should of course proceed to test equal volumes of serum and a fortyfold diluted normal acid. This mixture would, in the case of normal blood, almost to a certainty be alkaline. Supposing that it was alkaline, and that the reaction of the mixture of equal volumes of serum and thirty-fold diluted acid had proved to be acid, we should then proceed to mix in a clean watchglass equal volumes of a thirty-and a forty-fold diluted normal acid. We should tritrate our serum again with this thirtyfire-fold diluted normal acid. If we found that this quantity of acid just sufficed to neutralise the acidity of our sample of serum, it is obvious that the allalinity of our serum would be most simply expressed by the fraction $\frac{N}{35}$. For a serum which is exactly neutralised by an equal volume of a thirty-fivefold diluted normal acid would obviously correspond in alkalinity to a thirty-five-fold diluted normal solution of alkali.

In a futare communication I hope to draw attention to some results of therapeutic and diagnostic importance which have been obtained by the exploitation of this method of measuring the alkalinity of the blood. For the present it will suffice to put on record for the guidance of others who may be disposed to employ this method of measuring the alkalinity of the blood that the maximum value which has been found by this method for the alkalinity of the normal serum has been $\frac{N}{25}$. This result has been obtained only once. In the case of thirteen other normal persons the serum has been found to possess an alkalinity which has always varied between the values of $\frac{N}{30}$ and $\frac{N}{45}$. On the arerage the alkalinity of the serum has been equivalent to about $\frac{N}{35}$. Netley.

\section{A 3 hlitrot}

\section{HOSPITAL PRACTICE, BRITISH AND FOREIGN.}

Nulla autem est alia pro certo noscendi via, nisi quamplurimas et morborum et dissectionum historias, tum aliorum tum proprias collectas habere, et inter se comparare.-MorGaGNI De Sed. et Caus. Morb., lib. iv. Procmium.

\section{ROYAL FREE HOSPITAL.}

A CASE OF DILATATION OF THE STOMACH ASTOCIATED WITH PERIPHERAL NEURITIS.

(Under the care of Dr. WALTER CARR.)

A MAN, aged twenty-eight years, was admitted into the Royal Free Hospital on Nov. 20th, 1896. He said that since he was about sixteen years old he had been subject to occasional attacks of abdominal pain, lasting about twelve hours and not specially related to food. For some years these attacks were not attended by sickness, and the symptoms from which he suffered do nct suggest that he had a gastric ulcer; neither had he ever swallowed any corrosive fluid. Since 1890 the attacks of prin had recurred about every two months, had been more severe, and had been terminated by copious vomiting. Since about Chrisimas, 1895, they had become much more frequent, sometimes occurring two or three times a week, with very severe pain; exertion seemed to increase their frequency. The patient said that before the attacks his stomach felt very uncomfortable and he could feel it "working" (evidently the peristaltic movements). After the sickness he was much relieved for a time. In June, 1896, his legs had began to get weak, with much pain in the calf muscles, numbness and tingling in the legs and slightly in the hands; since August he had been unable to walk and had wasted rapidly. No history was obtained of any acute illness except influenza five years ago. He entirely denied any alcoholic excess, saying that he had rarely taken more than one or two glasses of beer a day. On admission the patient was seen to be a well-built man, about $5 \mathrm{ft} .11$ in. in height, but greatly emaciated; his weight was 9 st. $7 \mathrm{lb}$. He had not been sick for a week, but the day after admission he brought up $7 \frac{1}{2}$ pints, and romited from 1 pint to $2 \frac{1}{2}$ pints nearly every day for the next week he also brought up much flatus. The romit contained abundant sarcinæ, but no blood. The tongue was clean, the appetite fairly good, and the bowels acted nearly every day. On examination of the abdomen peristalsis was frequently visible, starting in the left hypochondrium and passing across the umbilical region to the right. On palpation no tumour, thickening, or resistance could be detected in the neighbourhood of the pylorus or elsewhere. The stomach was distended by giving bicarbonate of potassium and citric acid, and was found to be greatly dilated, reaching well below the umbilicus, though the exact limits could not be defined. The liver dulness was normal. In regard to the nervous syctem there was no complete paralysis of any limb, but his $1 \mathrm{kgg}$ were very weak, especially the left, and he was unable to extend the left toes. He could only walk very feebly, requiring a great deal of support. He could just stand alone, but not when the eyes were shut. No other evidence of ataxy was detected. All the muscles of the legs were much wasted. In the upper limbs wasting was especially marked in the supra- and infra. spinati and the interossei. In the muscles below the knees no reaction was obtained to faradaism, but all the muscles reacted to the galvanic current. He complained of tingling and numbness in the legs, and the calf muscles were very tender. There was very slight impairment of sensibility below the knees, chiefly about the feet. No girdle sensation was present. The kneejerks and plantar reflexes were absent. There was no loss of sexual power or of control over the sphincters. His pupils were normal and no ophthalmoscopic changes were found. His chest was healthy. The temperature was normal throughout his stay in the hospital. The urine contained no albumin or sugar.

The patient was kept in bed and carefully dieted; he was given hot water to drink before each meal, little or no fluid being allowed with the food; strychnine 